\title{
Effects of dissipating gas drag on planetesimal accretion in binary systems
}

\author{
Ji-Wei Xie and Ji-Lin Zhou \\ Department of Astronomy, Nanjing University, China \\ email: xjw0809@163.com; zhouj1@nju.edu.cn
}

\begin{abstract}
We numerically investigate the conditions for planetesimal accretion in the circumprimary disk under the perturbing presence of a companion star, with focus on the $\gamma$ Cephei system. Gas drag is included with a dissipating time scale of $10^{5}$ years. We show at the beginning(within $10^{3} \sim 10^{4}$ years), gas drag damps the $\Delta V$ between planetesimals of same sizes and increases $\triangle V$ between planetesimals of different sizes. However, after increasing to high values $(300 \sim 800 \mathrm{~m} / \mathrm{s})$, we find the $\triangle V$ between bodies of different sizes decrease to very low values (below $10 \mathrm{~m} / \mathrm{s}$ ) in a few $10^{5}$ yrs (depending on the gas-dissipating time scale $T_{\text {dam }}$, radial size $R_{p}$ and semi-major axis $a_{p}$ of planetesimals). Hence, the high $\triangle V$ is somewhat short-lived, and runaway accretion can be turned on later. We conclude that the conditions for planetary formation in binary systems (even close binary systems) are much better than what we expected before.
\end{abstract}

Keywords. planetary formation, accretion, planetesimals, binary stars

\section{Introduction}

Because of the increasing number of discovered planets in binary systems and the belief that a majority of solar type stars reside in binary or multiple systems, problem of planetary formation in binary systems becomes a crucial one. Most of the discovered binary systems are S-type systems(e.g. $\gamma$ Cephei system, see Hatzes et al. 2003) in which planets orbit the primary star and with a companion star surrounding them on an outer orbit. According to the classical planetary formation scenario, planets form in a protoplanetary disk of gas and dust orbiting a protostar. The formation process is usually treated in three stages: [stage I] formation of kilometers-size plantesimals $\left(10^{18}-10^{22} \mathrm{~g}\right)$ from dust accretion; [stage II] accretion of plantesimals to planetary embryos $\left(10^{25}-10^{26} \mathrm{~g}\right.$, Mercury- to Mars-size) in $\sim 10^{5}$ years, depending on the initial disk density; [stage III] giant impacts between embryos, producing full-size $\sim 10^{26}$ to $\sim 10^{27}$ g terrestrial planets in $\sim 10^{7}-10^{8}$ years. Here we focus on the stage II to see the influence of the companion on the planetesimal accretion.

The companion star, especially when it is in a close orbit with a high eccentricity, may prevent planetary formation because the companion reduces the size of the accretion disk, and it excites high relative velocities between colliding planetesimals. The relative impact velocity $(\triangle V)$ is a critical parameter which determines whether accretion or erosion dominates the planetesimal collisional evolution. Due to the perturbation by the companion, $\triangle V$ may exceed the planetesimal escape velocities $\left(V_{e s c} \sim 10 \times\left(R_{p} / k m\right) m s^{-1}\right)$, thus preventing runaway accretion, or even the threshold velocity $\left(V_{\text {ero }} \sim b \times V_{\text {esc }}\right)$ for which erosion dominates accretion, where $b$ is a dimensionless coefficient of $2 \sim 5$ roughly depending on the prescription on collision. However, the presence of gas drag does not only damp the secular perturbation, but it also forces a strong periastron alignment of planetesimal orbits. This alignment significantly reduces $\triangle V$, favoring the accretion process(Marzari \& Scholl 2000). Nevertheless, the alignment forced by the gas drag induces 
Table 1. initial parameters for runs

\begin{tabular}{lr}
\hline semi-major axis of companion & $a_{B}=18.5 \pm 1.1 \mathrm{AU}$ \\
eccentricity of binary & $e_{B}=0.361 \pm 0.023$ \\
mass of primary & $M_{A}=1.6 M_{\odot}$ \\
mass of companion & $M_{B}=0.4 M_{\odot}$ \\
physical radius of planetesimals & $R_{p}=2.5,5,15,50 \mathrm{~km}$ \\
initial eccentricities of planetesimals & $0<e<10^{-3}$ \\
initial inclinations of planetesimals & $0<i<5.10^{-4}$ \\
planetesimal density & $3 \mathrm{~g} \cdot \mathrm{cm}^{-3}$ \\
initial gas density at 2AU & $\rho_{g 20}=2.10^{-9} \mathrm{~g} \cdot \mathrm{cm}^{-3}$ \\
initial gas density radial profile & $\rho_{g} \propto \mathrm{r}^{-2.75}$ \\
gas-dissipating time scale & $T_{\text {damp }}=10^{5}$ years \\
\hline
\end{tabular}

another problem. Since the alignment is size-dependent, it can only reduce $\triangle V$ between planetesimals of same size, and at the same time it increases $\triangle V$ between planetesimals of different sizes. Thebault et al. (2006) found that the differential orbital alignment was very efficient, leading to a significant $\triangle V$ increase for any departure from the exact equalsize condition $\left(R_{1}=R_{2}\right.$, where $R_{1}$ and $R_{2}$ are the radial sizes of two colliding bodies). We reinvestigate the effects of gas drag on relative velocities $\triangle V$ among a swarm of planetesimals in $\gamma$ Cephei system(as our model system, and the mechanism can be applied to other binary systems). We will show that the presence of a dissipating gas can induce a $\triangle V$ decrease between planetesimals of different sizes in a timescale $(2 \sim 5) \times T_{\text {damp }}$ (gasdissipating timescale). After $(2 \sim 5) \times T_{\text {damp }}$ the $\triangle V$ between planetesimals of different sizes will reach very low values, allowing runaway accretion.

\section{Numerical model and method}

Here we adopt the parameters listed in table 1 . We concentrate on four sizes planetesimals $\left(R_{p}=2.5,5,15,50 \mathrm{~km}\right)$. The relative velocity $\triangle V(2.5,5)$ between bodies of $R_{p}=2.5 \mathrm{~km}$ and $R_{p}=5 \mathrm{~km}$ can be representative for small planetesimals, and so does $\triangle V(15,50)$ for large planetesimals (Thebault et al. 2006).

Following Weidenschilling and Davis (1985), the gas drag can be expressed as:

$$
\mathbf{F}=-K v \mathbf{v}
$$

where $\mathbf{F}$ is the force per unit mass, $\mathbf{v}$ the relative velocity between planetesimal and gas, $v$ the velocity modulus, and $K$ is the drag parameter defined as:

$$
K=\frac{3 \rho_{g 0} C_{d}}{8 \rho_{p} R_{p}} \exp \left(-t / T_{d a m p}\right)
$$

where $\rho_{g 0}$ is the initial gas density, $\rho_{p}$ and $R_{p}$ the planetesimal density and radius, respectively. $C_{d}$ is a dimensionless coefficient related to the shape of the $\operatorname{body}(\simeq 0.4$ for spherical bodies). The exp function is used to include the dissipation of gas with a 


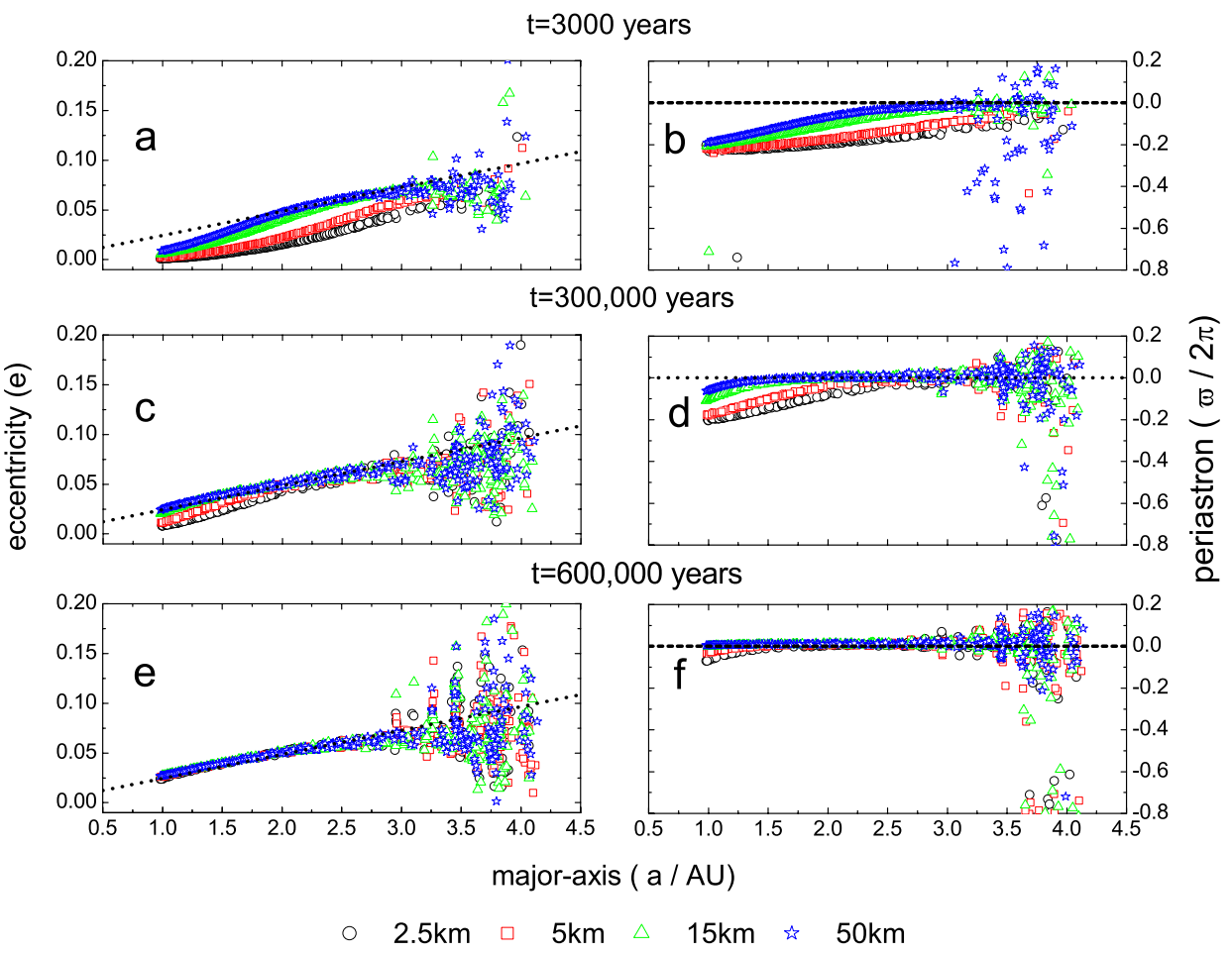

Figure 1. Distributions of planetesimal eccentricities and periastrons vs. semi-major axes at three different epoches. Bodies of different sizes are plotted in different styles. The dashed lines which are in the 3 left panels and 3 right ones denote $e_{f}$ (forced eccentricity) and $\varpi=0$ (which means the planetesimal periastrons $w_{p}=w_{f}$ the forced periastron), respectively.

damping time scale $T_{\text {damp }}$. As shown in Eq.(2), the gas drag force is size-dependent $(\propto$ $\left.1 / R_{p}\right)$.

It has been shown by Gladman (1993) that two isolated planetesimals will eventually collide if they are within a critical distance $2.4\left(\mu_{1}+\mu_{2}\right)^{1 / 3} a$, where $\mu_{1}, \mu_{2}$ and $a$ are the masses in solar units and average semi-major axis of two colliding planetesimals, respectively. We adopt it as the criterion of searching for impact, and calculate the $\triangle V$ for each impact. In all the runs, we used the fourth order Hermite integrator (Kokubo et al., 1998), including the gas drag force and the perturbation of companion. As gas drag also forces inward drift of planetesimals, we adopt the following boundary conditions: bodies whose major-axes are less than $R_{\text {in }}$ (greater than $R_{\text {out }}$ ), will be reset to $R_{\text {out }}\left(R_{\text {in }}\right)$, where $R_{\text {in }}$ and $R_{\text {out }}$ are inner and outer boundaries of planetesimal belt. In these resetting processes, only the semi-major axes of those bodies are changed, and other orbital elements are preserved.

\section{Result}

We first performed a simulation where 1000 planetesimals (4 equal-number groups: $R_{p}=2.5,5,15,50 \mathrm{~km}$, mutual interactions are neglected) were initially distributed 
between $1 \mathrm{AU}$ and $4 \mathrm{AU}$ from the primary. Fig. 1 shows the distributions of planetesimal eccentricities and periastrons vs. semi-major axes at different epoches. Beyond 3 AU, the distribution of planetesimal eccentricities and periastrons are random because the shorter period perturbations and mean motion resonances dominate. Hence, hereafter only planetesimals within $3 \mathrm{AU}$ are discussed. In Fig. 1a(b), within $3 \mathrm{AU}$ (hereafter), every eccentricity (periastron) reaches an equilibrium phased value at 30,000 years. This equilibrium phased value depends on the balance between the perturbation by the companion and the gas drag force. Due to the side-dependence of gas drag force, bodies of different sizes reach different equilibrium eccentricities (periastrons). In Fig. 1a(b) the four lines are associated with bodies of four kinds of sizes $\left(R_{p}=2.5,5,15,50 \mathrm{~km}\right)$. As the gas dissipates gradually, the equilibrium phased eccentricities (periastrons) move to larger values, but at the same time the differences in eccentricity (periastron) distributions among bodies of different sizes become smaller (see Fig. 1c(d)). After a long time (600,000 years, see Fig. 1e(f)), almost all eccentricities (periastrons) fix on $e_{f}\left(w_{f}\right)$. Hence, the differential phasing effect caused by the size-dependence of gas drag is reduced if the gas dissipation is included.

As shown in Fig. 1, dissipating gas drag reduces the effect of size-dependence of orbital alignment. Hence, we can expect a decrease in $\triangle V$ between bodies of different sizes. Fig. 2a shows the $\triangle V\left(R_{1}, R_{2}\right)$ as the function of time at $2 \mathrm{AU}$ from the primary star. Fig. 2b and Fig. 2c show the average eccentricity and periastron of bodies at $2 \mathrm{AU}$ as the functions of time. It is evident that a correlation(the larger differences in orbital elements, the larger value of $\triangle V$ ) exists between Fig. 2a and Fig. 2b(c). In this calculation, 1000 Planetesimals were initially distributed with major-axes between 1.5 and 3 AU. This ring is so wide that it includes most of the impacts that occur at $2 \mathrm{AU}$ (not semi-major axis of planetesimal orbit but distance from the center star). Although some bodies with
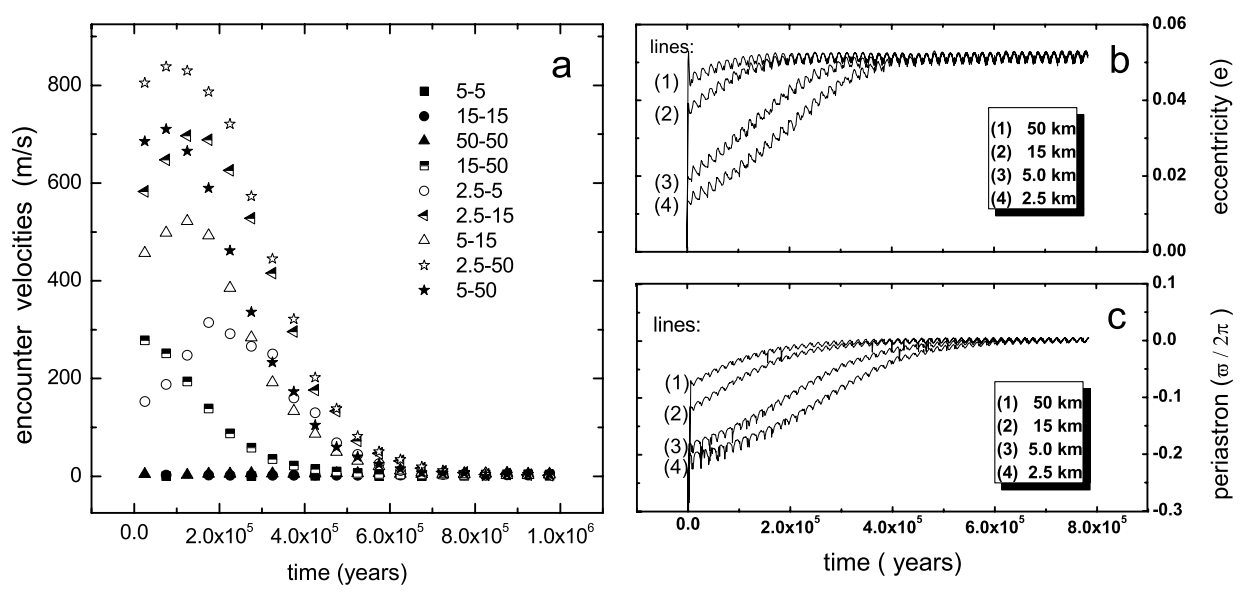

Figure 2. Correlations between $\triangle V$ and distributions of orbital elements. (a)Average encounter velocities $\triangle V$ at $2 \mathrm{AU}$ from the primary star v.s. time in dissipating gas case. $\triangle V$ between bodies of different sizes are plotted in different styles. (b) and (c): Average eccentricity and periastron $(\varpi)$ at $2 \mathrm{AU}$ from the primary v.s. time in dissipating gas case. Bodies of different sizes are plotted in different styles 
high eccentricities on outer orbits $(\mathrm{a}>3 \mathrm{AU})$ can cross the orbits of bodies near $2 \mathrm{AU}$ region, the rate of this kind impact is much lower because of the longer orbital periods and smaller number of planetesimals in outer region. Hence, a initial planetesimal belt between 1.5 and 3.0 $\mathrm{AU}$ is reasonable for tracing the $\triangle V$ at $2 \mathrm{AU}$.

From Fig. 2a, it appears that the $\triangle V$ between bodies of equal-side are always small because of orbital alignment. However, the $\triangle V$ between bodies of different sizes first increase to high values quickly(e.g. $300 \sim 800 \mathrm{~m} / \mathrm{s}$ ). Then each of them experiences a relatively slow decrease. After about $5 \times 10^{5}$ years they all stay steadily at low values (below $10 \mathrm{~m} / \mathrm{s}$ ). These low $\triangle V$ favor planetesimal accretion and even runaway growth. The time scale for $\triangle V$ decreasing depends on the gas damping time scale $T_{\text {damp }}$, body size $R_{p}$ and semi-major axis of body $a_{p}$. From Fig. 2a, $\triangle V\left(R_{1}, R_{2}\right)$ with $R_{1}$ or $R_{2}$ below $5 \mathrm{~km}$ decrease to low values within $\sim 5 \times T_{\text {damp }}$, but only $\sim 2 \times T_{\text {damp }}$ is needed for $\triangle V$ $\left(R_{1}, R_{2}\right)$ with $R_{1}$ and $R_{2}$ both greater than $15 \mathrm{~km}$. Furthermore, from Fig. $1 \mathrm{~b}(\mathrm{c})$, bodies at outer region converge their eccentricities (periastrons) faster than inner bodies. For this reason, we find $\triangle V\left(R_{1}\right.$ or $\left.R_{2}<5 \mathrm{~km}\right)$ decreases to and stays steadily on $30 \mathrm{~m} / \mathrm{s}$ $(100 \mathrm{~m} / \mathrm{s})$ within $\sim 4(3) \times T_{\text {damp }}$ for bodies at 2.5 (3.0) AU. The larger equilibrium value of $\triangle V$ at outer region is due to the larger perturbation there.

\section{Conclusion}

The previous results show that $\triangle V$ depends on the delicate balancing between gas drag and secular perturbation by the companion. Bodies of different sizes reach different equilibrium values in eccentricities and periastrons. This is called size-dependence of orbital alignment. Its effect is very efficient and induces a significant $\Delta V$ increase between bodies of different sizes. Based on these facts, Thebault et al. (2006) investigated $\triangle V$ for binary systems of various orbital parameters. They found on the region at a distance of $1 \mathrm{AU}$ from the primary, $\triangle V$ is always high preventing runaway accretion, except in a narrow $e_{b} \simeq 0$ domain. In this paper, focusing on the $\gamma$ Cephei system, we adopt a fast dissipating and massive(initial density is $10 \mathrm{MMS}$ ) gas disk model, which is possible for some close binary systems. We also find a significant increase of $\triangle V$ between bodies of different sizes caused by size-dependence of orbital alignment, but at the same time this high $\triangle V$ decreases to a very low value in about $(2 \sim 5) \times T_{\text {damp }}$. Notice that the $\triangle V$ will not increase again even when the gas is dissipated totally. After $(2 \sim 5) \times T_{\text {damp }}$, the $\triangle V$ is low enough for runaway accretion, and there is enough gas $(0.1 \sim 1.0 M M S)$ left for gas giant formation.

\section{Acknowledgments}

We thank W. Kley for useful discussions and suggestions. This work is supported by NSFC(10778603), National Basic Research Program of China(2007CB4800).

\section{References}

Desidera, S. \& Barbieri, M. 2007, A\&A, 462, 345

Eggenberger, A., Udry, S., \& Mayor, M. 2004, A\&A, 417, 353

Gladman, B., 1993, Icarus, 106, 247

Hatzes, A.P., Cochran, W. D., Endl, M., McArthur, B., Paulson, D., Walker, G. A. H., Campbell, B., Yang, S., 2003, ApJ, 599, 1383

Hayashi, C., 1981, PthPS, 70, 35

Kokubo, E., Yoshinaga K., \& Makino, J. 1998, MNRAS, 297, 1067

Marzari, F. \& Scholl, H. 2000, ApJ, 543, 328 
Thebault, P., Marzari, F., Scholl, H., Turrini,D., Barbieri,M.,2004,A\&A,427,1097

Thebault, P., Marzari, F., Scholl, H., 2006, Icarus, 183, 193

Tsukamoto, Y. \& Makino, J. 2007, Formation of protoplanets from massive planetesimals in binary systems, eprint arXiv: astro-ph/07072928

Weidenschilling, S. J. \& Davis, D. R., 1985, Icarus, 62, 16 